\title{
The Diabetes Poland Working Group on Psychology - past, present and questions about the future
}

The letter refers to the paper that has been published in "Clinical Diabetology" 6/2016: Foryciarz K, Obrębski M, Kokoszka A. Results of the second Diabetes Attitudes, Wishes and Needs (DAWN2) study: Overview of the most important results from the Polish perspective. Clin Diabetol 2016; 5, 6: 185-194. DOI: 10.5603/DK.2016.0032.

A review of the most important results of the second international DAWN (Diabetes, Attitudes, Wished and Needs) study is an opportunity to reflect on the work of the Diabetes Poland (DP) Working Group on Psychology. I have been its chair since its foundation in 2007. In the first years, there were psychological seminars on the occasion of major diabetic conferences, attended by 8 to 20 people. Attempts at scientific cooperation have been made, which resulted in presentation of its preliminary results during the annual meeting of the Psychosocial Aspects of Diabetes Study Group of the European Association for the Study of Diabetes (EASD). Later, psychological sessions were organized at DP congresses, most recently in Lodz where my colleagues were increasingly involved.

In recent years, fewer and fewer psychologists have been involved in these conferences and the Working Group's activities have been confined to the activities of my team in the 2nd Department of Psychiatry of the Medical University of Warsaw. We participate in two major international projects: the DAWN 2 Study and
INTERPRET-DD International Prevalence and Treatment of Diabetes and Depression. I am a co-author of the publications presenting the results of these research programs and several other studies on the psychological problems associated with diabetes. The clinic I supervise is preparing 6 doctoral theses in psychodiabetology based on current research.

Gradually, my contacts with psychologists dealing with psychotherapeutic interventions for patients with diabetes and physicians interested in diabetes psychology have disappeared. In this situation, it seems reasonable to ask about:

- the need to intensify the work of the Working Group on Psychology;

- decision to dissolve the Working Group;

- or change the chairman of the Working Group and a "new opening".

I will highly appreciate your opinions and comments on these issues. Please kindly send them to my email: andrzej.kokoszka@wum.edu.pl

Andrzej Kokoszka 